\title{
Experimental Evaluation of Turbidity Impact on the Fluence Rate Distribution in a UV Reactor Using a Microfluorescent Silica Detector
}

\author{
Mengkai Li, ${ }^{\dagger, \ddagger}$ Wentao Li, ${ }^{\ddagger}$ Dong Wen, Zhimin Qiang, ${ }^{*},{ }^{\ddagger}$ and Ernest R. Blatchley, III*, ${ }^{*}, \S_{\odot}$ \\ ${ }^{\dagger}$ Lyles School of Civil Engineering, Purdue University, West Lafayette, Indiana 47907, United States \\ ${ }^{\ddagger}$ Key Laboratory of Drinking Water Science and Technology, Research Center for Eco-Environmental Sciences, University of Chinese \\ Academy of Sciences, Chinese Academy of Sciences, 18 Shuang-qing Road, Beijing 100085, China \\ ${ }^{\S}$ Division of Environmental \& Ecological Engineering, Purdue University, West Lafayette, Indiana 47907, United States
}

Supporting Information

\begin{abstract}
Turbidity is a common parameter used to assess particle concentration in water using visible light. However, the fact that particles play multiple roles (e.g., scattering, refraction, and reflection) in influencing the optical properties of aqueous suspensions complicates examinations of their effects on ultraviolet (UV) photoreactor performance. To address this issue, UV fluence rate (FR) distributions in a photoreactor containing various particle suspensions $\left(\mathrm{SiO}_{2}, \mathrm{MgO}\right.$, and $\left.\mathrm{TiO}_{2}\right)$ were measured using a microfluorescent silica detector (MFSD). Reflectance of solid particles, as well as transmittance and scattering properties of the suspensions were characterized at UV, visible, and infrared (IR) wavelengths. The results of these measurements indicated that the optical properties of all three particle types were similar at visible and IR wavelengths, but obvious differences were evident in the UV range. The FR results indicated that for turbidity associated with $\mathrm{SiO}_{2}$ and $\mathrm{MgO}$ suspensions, the weighted average FR (WAFR) increased relative to deionized water. These increases were attributed to low particle photon absorption and strong scattering. In contrast, the WAFR values decreased with increasing turbidity for $\mathrm{TiO}_{2}$ suspensions because of their high particle photon absorption and low scattering potential. The findings also indicate that measurements of scattering and transmittance at $\mathrm{UV}$ wavelengths can be used to quantify the effects of turbidity on UV FR distributions.
\end{abstract}

\section{INTRODUCTION}

In recent years, ultraviolet (UV) technology has been used increasingly for disinfection, photolysis, and advanced oxidation processes in water and wastewater treatment. ${ }^{1,2}$ The performance of UV photoreactors is governed by fluence (dose) delivery, in particular the fluence distribution. Numerical models have been widely applied to calculate fluence delivery, generally based on integrated application of submodels to simulate the optical field (i.e., fluence rate (FR) distribution) and fluid mechanics, usually by application of computational fluid dynamics software., An important component of accurate simulations of fluence delivery is an accurate description of the FR distribution. ${ }^{5-7}$

Several mathematical models have been developed for simulation of FR distributions in UV reactors, including the multiple point source summation (MPSS), ${ }^{8}$ line source integration (LSI), ${ }^{9}$ and extense source with volumetric emission (ESVE) models. ${ }^{10}$ Meanwhile, hardware and analytical methods to measure (local) FR have been developed to validate optical field models. ${ }^{11,12}$ In general, FR models are effective for quantification of the effects of dissolved compounds that may absorb UV radiation. ${ }^{13,14}$ The fundamental optical behavior of these dissolved compounds (i.e., absorbance) can be accurately measured using a spectrophotometer based on the LambertBeer Law.
When UV radiation is imposed on particle suspensions, photon absorption and scattering (or reflection) by suspended particles will occur. This complex optical behavior is difficult to simulate using existing models, and has not been examined in a systematic manner, largely because of the dispersed nature of the suspended particles. In fact, absolute scattering does not induce photon energy consumption; scattered photons remain available for disinfection or photolysis, while absorbed photons are no longer available for reactions. ${ }^{15,16}$ Hence the LambertBeer law is insufficient for FR distribution simulation in the presence of particle suspensions, and measurements based on standard spectrophotometers do not allow independent characterization of the optical behavior of suspensions. Therefore, in the absence of numerical methods to account for the optical effects of suspended particles, experimental measurements of the FR field could be used to provide direct evidence of the effects of particles.

Turbidity measurements are commonly used to characterize the particle concentration in water. ${ }^{17,18}$ Despite the fact that turbidity measurements are generally based on visible radiation,

Received: May 30, 2017

Revised: October 23, 2017

Accepted: October 25, 2017

Published: October 25, 2017 
this commonly applied optical parameter has also been used to inform water quality assessments that are applied for performance analysis of UV photoreactors. At present, turbidity in water treatment is usually measured using an electronic turbidity meter, usually expressed in nephelometric turbidity units (NTUs). Multiple turbidity measurement methods have been approved by the United States Environmental Protection Agency (USEPA) and the International Organization for Standardization (ISO), including EPA Method 180.1, ISO7027, Great Lakes Instrument Method 2 (GLI 2), Hach Method 10133, etc. These methods are based on nephelometry, wherein a visible light beam and a photodetector are positioned to allow measurement of visible light scattered perpendicular to the incident beam.

To date, most studies of the effects of turbidity on UV FR fields have involved empirical investigations based on benchscale reactors. Passantino reported that clay turbidity up to 12 NTU does not have an obvious effect on MS2 inactivation. ${ }^{20}$ Amoah found that UV inactivation of $C$. parvum and Giardia(oo)cysts was not impacted by 10 NTU of natural turbidity. ${ }^{21}$ However, Malley observed that different suspended particles have different inhibition impacts on the performance of MS2 virus inactivation by UV. ${ }^{22}$ Templeton demonstrated that humic acid and activated sludge floc particles can protect MS2 coliphage and bacteriophage T4 from UV inactivation. ${ }^{23}$ Mamane et al. examined the effects of turbidity on the FR field using spherical actinometry and natural particles. ${ }^{24}$ Their work demonstrated that particles could yield increases in local FR, depending on the nature of the particles.

A micro fluorescent silica detector (MFSD) was developed for in situ measurement of FR distributions in UV photoreactors in our previous research. ${ }^{12,25}$ The key features of this detector include 360-degree response, high stability, fast response, water-resistance, and small volume; these features have allowed it to be used for the examination of a wide range of optical characteristics of UV photoreactors, including the effect of inner-wall reflection, ${ }^{25}$ polychromatic lamp reactor behavior, ${ }^{14}$ and multilamp reactor behavior. ${ }^{26}$ Therefore, it was hypothesized that this detector would be suitable for the examination of the turbidity effect in UV photoreactors. This work aimed to clarify, by use of the MFSD, the impact of turbidity on the FR distribution in a UV reactor.

A second hypothesis was that measurements of reflectance and scattering at the characteristic wavelength of the UV source would yield better indications of the effects of suspended particles on the FR field than traditional measurements of turbidity. With this in mind, three different substituted oxide powders (SOPs, namely $\mathrm{SiO}_{2}, \mathrm{MgO}$, and $\mathrm{TiO}_{2}$ ) with a range of reflection coefficients were examined. The optical properties of the solid particles and suspension were characterized independently, and then UV FR distributions in various particle suspensions at various turbidities were measured. This study provides experimental evidence of the effects of suspended particles on the FR field of a UV photoreactor. The results suggest promise for practical examination and monitoring of the particle effect on UV photoreactors used in water treatment.

\section{EXPERIMENTAL SECTION}

Chemicals and Analytical Methods. Three SOPs, namely $\mathrm{TiO}_{2}$ (R902, DuPont Co., particle diameter (PD) ca. $400 \mathrm{~nm}$ ), $\mathrm{SiO}_{2}$ (Cabot M-5, PD ca. $300 \mathrm{~nm}$ ), and $\mathrm{MgO}$ (Sinopharm Chemical Reagent Co., PD ca. $1000 \mathrm{~nm}$, China), were used to prepare particle suspensions with various turbidities. The reflection coefficients of the solid SOPs were measured at wavelengths of 254,600 , and $800 \mathrm{~nm}$ using a spectrophotometer (U-3900, Hitachi, Japan) equipped with an integrating sphere attachment; reported reflectance values were the sum of specular and diffuse measurements. Powders of all three SOPs were compressed into tablet mold individually for the reflection coefficient measurement. ${ }^{27}$ Because of higher reflection coefficients of the $\mathrm{MgO}$ powder than that of the initial standard plate of this spectrophotometer (i.e., aluminum-evaporated plane) in the wavelength range of $240-280 \mathrm{~nm}, \mathrm{MgO}$ powder was used as the standard plate in this study. Therefore, reported reflection coefficients are relative to those of $\mathrm{MgO}$.

Aqueous suspensions of SOPs were prepared with deionized (DI) water. A HACH $2100 \mathrm{~N}$ turbidity meter was employed to measure the turbidity of aqueous particle suspensions. Precision of $0.01 \mathrm{NTU}$ was reported by the manufacturer for the range of 0-1000 NTU. The turbidity meter was equipped with a stable halogen-filled tungsten filament lamp (emission spectrum spanned the wavelength range $400-1800 \mathrm{~nm}$ ) to meet the reporting requirements of EPA Method 180.1. This method is based on the principles of nephelometry, and the photodetector, whose response spectral peak was reported to be between 400-600 nm, received light scattered perpendicular to the incident light path. Additionally, because the turbidity meter did not allow measurements based on radiation in the UV portion of the electromagnetic spectrum, a fluorescence spectrophotometer (Cary Eclipse, Varian, Australia) with a 1 $\mathrm{cm}$ path-length cell was utilized to investigate the scattering behavior of each SOP suspension at specific wavelengths in the UVC $(254 \mathrm{~nm})$, visible $(600 \mathrm{~nm})$, and IR (800 nm) ranges. In the fluorescence spectrophotometer, as with the turbidity meter, the excitation and emission beams are perpendicular to each other. For these measurements, the excitation and emission wavelengths were both set to the same value. Hence, the receiving "fluorescence" signal was used to represent the scattering property of the test suspension at each of the chosen wavelengths. Note that in common spectrofluorometric applications, the emission wavelength is set to a longer value than the excitation wavelength, hence the excited fluorescent signal of the suspension cannot interfere the scattering property test. In addition, absorbance at each of the three characteristic wavelengths (i.e., 254,600 , and $800 \mathrm{~nm}$ ) of each SOP suspension was measured with a spectrometer (UV2600, Shimadzu, Japan) in a $1 \mathrm{~cm}$ path-length cell.

FR Distribution Measurement Platform. Figure 1 represents a schematic diagram of the experimental platform for measuring the FR distributions in a single-lamp UV photoreactor. A cylindrical MFSD (1.0 mm length $\times 0.3 \mathrm{~mm}$ outside diameter), whose fluorescence was received by a BPW 34 B silicon PIN photodiode, and amplified and displayed by a multimeter, was used as the FR measurement detector in this study. A cylindrical quartz-wall UV reactor (inner diameter = $190 \mathrm{~mm}$ and inner length $=1000 \mathrm{~mm}$ ) wrapped in a black cotton cloth was used; UV reflectance from the air/quartz/air interfaces of this reactor had previously been estimated at approximately $8 \% .{ }^{24}$ A single low pressure mercury $\mathrm{Hg}$ lamp (GL Type, Xiashi Wanhua Co., China) with a quartz sleeve (radius $=11.5 \mathrm{~mm}$ ) was housed in the center of the cylindrical quartz photoreactor. The lamp parameters were: arc length = $297 \mathrm{~mm}$; total output power $16 \mathrm{~W}$; UVC efficiency at $254 \mathrm{~nm}=$ $26 \%$. 


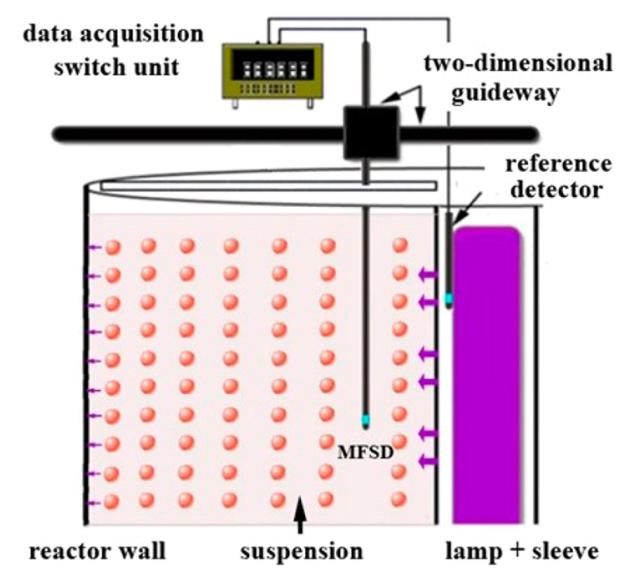

Figure 1. Schematic diagram of the experimental platform for measuring the FR distributions in a UV photoreactor.

The MFSD was fixed onto a two-dimensional guideway with its vertical axis oriented parallel to the lamp axis (Figure 1). This configuration allowed the detector to move either parallel (displacement precision of $1 \mathrm{~mm}$ ) or perpendicular (displacement precision of $0.01 \mathrm{~mm}$ ) to the lamp axis to measure the axial or radial FR distribution, respectively. The calibration process of the MFSD at $254 \mathrm{~nm}$ has been described previously. ${ }^{12}$ A second MFSD, used as a reference detector, was inserted into the gap between the lamp and the sleeve to monitor lamp output fluctuations. Water temperature was controlled by a recirculator with precision of $0.5{ }^{\circ} \mathrm{C}$.

FR Distribution Measurement process. The FR distributions in the reactor were measured for each of several particle suspensions. Note that for mixed suspensions of $\mathrm{SiO}_{2}$ and $\mathrm{TiO}_{2}$, the $\mathrm{SiO}_{2}$ powder was first added to reach the turbidity requirement (e.g., $5.0 \mathrm{NTU}$ for the $\mathrm{SiO}_{2} 50 \%$ $\mathrm{TiO}_{2} 50 \%$ case with $10.0 \mathrm{NTU}$ ) and then the $\mathrm{TiO}_{2}$ powder was added to reach the final turbidity requirement (e.g., 10.0 NTU for the $\mathrm{SiO}_{2} 50 \%-\mathrm{TiO}_{2} 50 \%$ case with $10.0 \mathrm{NTU}$ ).

For each suspension, the test MFSD was placed parallel to the UV lamp at radial distances of 5.00 and $60.00 \mathrm{~mm}$ away from the quartz sleeve. For each fixed radial distance, the detector was moved parallel to the lamp axis to measure the axial FR distribution in the UV reactor. Then the detector was moved perpendicular to the lamp axis at the central crosssection of the lamp to measure the radial FR distribution. The detector was maintained at each test point for $1 \mathrm{~min}$ to allow a data acquisition switch unit (34971A, Agilent Co.) to collect 30 readings from the test MFSD. For each location, the last 10 readings were recorded and averaged. The final result for each test location was divided by the corresponding reading of the reference detector to correct any potential error induced by the lamp output fluctuation.

\section{RESULTS AND DISCUSSION}

Reflection Coefficients of Various Solid Particles. Table 1 lists the reflection coefficients in the UVC region (i.e., $254 \mathrm{~nm}$ ) and visible/IR region (i.e., 600 and $800 \mathrm{~nm}$ ) of the three solid SOPs (i.e., $\mathrm{MgO}, \mathrm{TiO}_{2}$ and $\mathrm{SiO}_{2}$ ) that were compressed into plates. Because $\mathrm{MgO}$ was used as the reference standard for reflection in this study, reflection coefficients for this material at all wavelengths were reported as 1.000 . In the visible/IR light region, all three SOPs displayed similar and high reflectance, while at $254 \mathrm{~nm}$ the reflection coefficient of $\mathrm{TiO}_{2}$ was substantially lower than other two SOPs. These
Table 1. Relative Reflection Coefficients of Various Materials at Different Wavelengths. At Each Wavelength, The Reflection Coefficient Was Normalized Relative to the Value Recorded for $\mathrm{MgO}$

$\begin{array}{cccc}\text { wavelength }(\mathrm{nm}) & \mathrm{MgO} & \mathrm{TiO}_{2} & \mathrm{SiO}_{2} \\ 254 & 1.000 & 0.135 & 0.849 \\ 600 & 1.000 & 0.929 & 0.947 \\ 800 & 1.000 & 0.916 & 0.954\end{array}$

results indicate that the all three SOPs may have similar optical behavior in the visible/IR region; however, the low UV reflection coefficient of $\mathrm{TiO}_{2}$ may be expected to yield a different UV FR distribution as compared to the other two SOP suspensions. It should be noted that the $\mathrm{TiO}_{2}$ particles selected for use in this study were pigment grade, which often contain other metal oxides (e.g., $\mathrm{SiO}_{2}, \mathrm{Al}_{2} \mathrm{O}_{2}, \mathrm{ZrO}_{2}$ ) as surface modifications. These surface impurities can have profound effects on the optical behavior of $\mathrm{TiO}_{2}$ particles. As such, the optical characteristics of these $\mathrm{TiO}_{2}$ particles may be different than those of other $\mathrm{TiO}_{2}$ particles.

Scattering Properties of Various Suspensions. Measurements of UVT values at $600 \mathrm{~nm}$ as a function of the turbidity for various SOP suspensions are shown in Figure 2a.

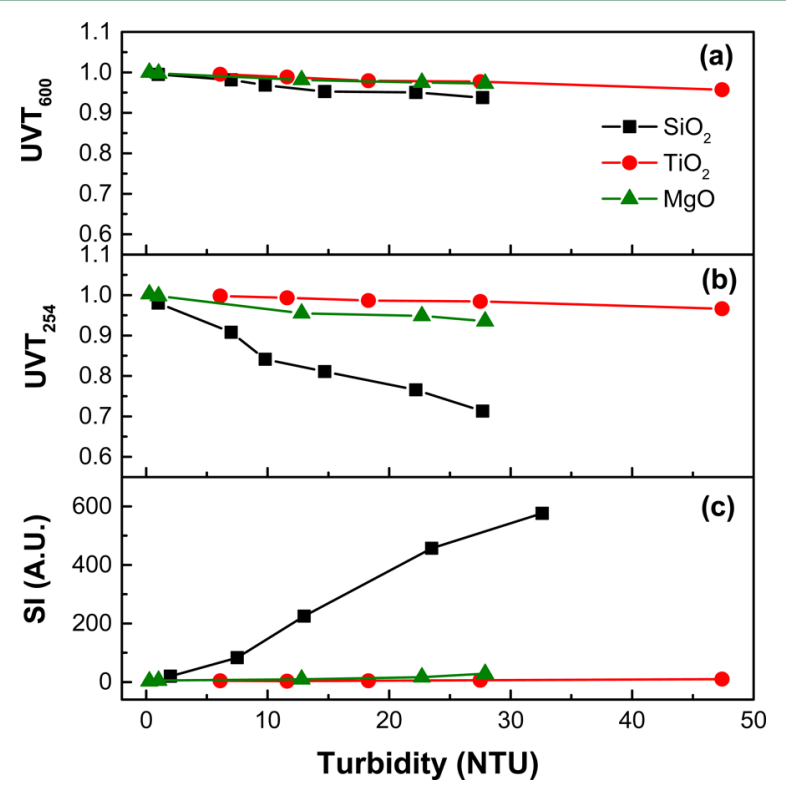

Figure 2. UVT at $600 \mathrm{~nm}$ (a), UVT at $254 \mathrm{~nm}$ (b), and scattering intensity (SI) at $254 \mathrm{~nm}$ (c) as a function of turbidity. All tests were repeated three times and all values of relative standard derivation were lower than $10 \%$.

Because of their similar reflection coefficients at $600 \mathrm{~nm}$, all three SOPs displayed similar relationships between $\mathrm{UVT}_{600}$ and turbidity. However, the particle suspensions did not display the same trends for $\mathrm{UVT}_{254}$ vs NTU (Figure 2b) and scattering intensity (SI) at $254 \mathrm{~nm}$, measured by the fluorescent spectrometer and defined by the strength of the signal scattered at an angle perpendicular to the incident beam (Figure 2c). A high particle concentration translates to high turbidity of a suspension; therefore, as turbidity increases it is reasonable to expect a decrease in $\mathrm{UVT}_{254}$ and a corresponding increase in SI. However, among the three SOP suspensions, the $\mathrm{SiO}_{2}$ suspension demonstrated the fastest decrease of $\mathrm{UVT}_{254}$ and the fastest increase of $\mathrm{SI}$, while the $\mathrm{TiO}_{2}$ suspension 
demonstrated opposite trends. These results suggest that these two parameters are complementary. For the measurements in the spectrometer and fluorescent spectrometer, incident UV photons will be scattered by an SOP suspension in the test cell, which would decrease the rate at which photons are received by the sensor oriented in the incident direction (i.e., $\mathrm{UVT}_{254}$ ) and increase the rate at which photons are received by the sensor oriented perpendicular to the incident direction (i.e., SI). Therefore, these two parameters could be used to evaluate the scattering property of the suspension, and in this study the $\mathrm{SiO}_{2}$ and $\mathrm{TiO}_{2}$ suspensions displayed the strongest and weakest scattering ability, respectively.

Note that the results of $\mathrm{UVT}_{254}$ and $\mathrm{SI}_{254}$ for the suspensions did not absolutely agree with the relative reflection coefficient measurements reported at $254 \mathrm{~nm}$ in Table 1 . We hypothesize that this is attributable to the fact that the scattering behavior of particles will depend on several factors, including particle size, particle shape, particle concentration, and chemical composition. The reflection coefficient data in presented in Table 1 only express the effects of chemical composition. By contrast, the scattering property test (i.e., Figures $2 b$ and $2 c$ ) is an integral characterization for the suspension, providing a measure of optical (scattering) behavior that accounts for all of the factors listed above.

Radial FR Distribution in Various Particle Suspensions. Figure 3 illustrates the measured radial FR distributions

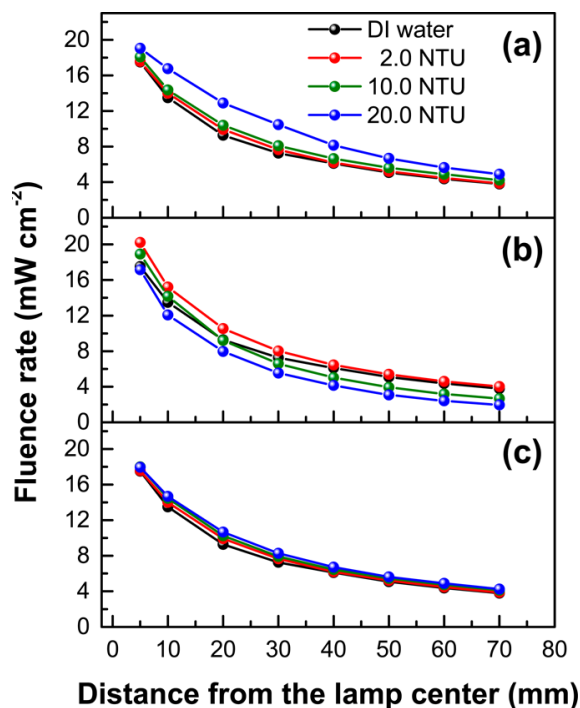

Figure 3. Radial FR distributions with various turbidities in (a) $\mathrm{SiO}_{2}$ suspension, (b) $\mathrm{TiO}_{2}$ suspension, and (c) $\mathrm{MgO}$ suspension.

in the central cross-section of the UV reactor filled with DI water, as well as the $\mathrm{SiO}_{2}, \mathrm{TiO}_{2}$, and $\mathrm{MgO}$ suspensions with various turbidities. When the test MFSD was moved from 10 to $70 \mathrm{~mm}$ from the sleeve surface, the FR decreased for all conditions. For the $\mathrm{SiO}_{2}$ suspension (i.e., Figure 3a), the local measurements of FR for all values of turbidity were higher than the corresponding measurements in DI water. As turbidity increased, local FR values also increased. These results imply that $\mathrm{SiO}_{2}$ particles in water will strongly reflect or redirect UV photons in various directions, thereby allowing photons to travel along a longer optical path than they would in the absence of these particles. In theory, this provides more opportunities for photons to interact with photochemical or photophysical targets in the system, such as a suspended microorganisms, absorbing dissolved chemicals, or an MFSD detector. Therefore, the presence of suspended, reflective particles could promote photoreactor efficiency. Similar results have been reported previously with an aluminum clay suspension. ${ }^{23}$

Similar results were also observed with the $\mathrm{MgO}$ suspensions shown in Figure $3 \mathrm{~b}$. The $\mathrm{FR}$ distribution in the $\mathrm{MgO}$ suspension increased with increasing turbidity and was consistently higher than with DI water. However, for the $\mathrm{TiO}_{2}$ suspensions, a consistent increase of FR, relative to DI water, was evident only for the 2.0 NTU suspension. FR enhancement was more evident in the near-lamp region than for locations more distant from the lamp. As turbidity increased from 2.0 to $20.0 \mathrm{NTU}$, the FR distribution dropped. This was consistent with the relatively strong absorption at $254 \mathrm{~nm}$ (i.e., low refection coefficient) and low scattering ability of the $\mathrm{TiO}_{2}$ particles, as compared to the other two SOPs. As the MFSD approached the reactor wall, photons had more opportunities to be absorbed by the $\mathrm{TiO}_{2}$ particles and were less likely to be scattered than with the other two particle suspensions.

Axial FR Distribution in Various Particle Suspensions. The axial FR distributions in the far-lamp region of the test UV reactor $(60 \mathrm{~mm}$ from the sleeve surface) for each SOP suspension are illustrated in Figure 4. The FR decreased as the

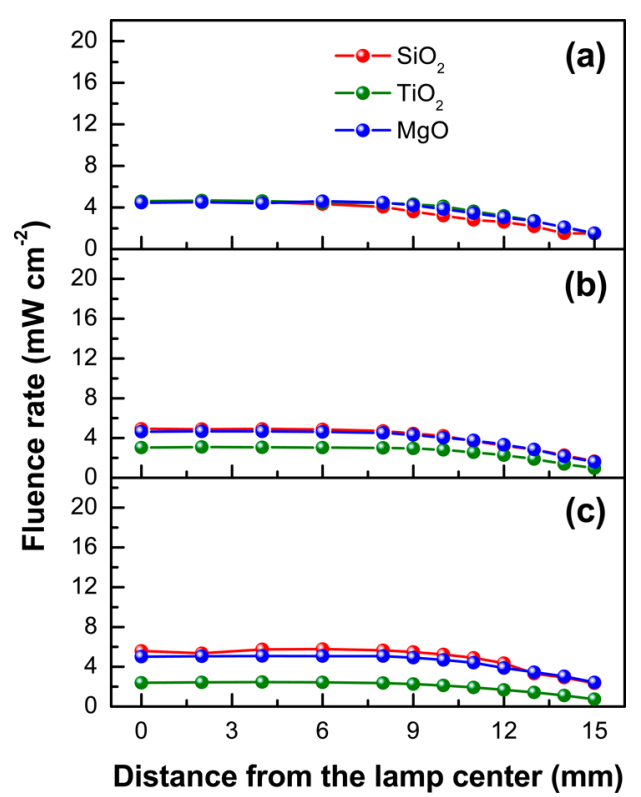

Figure 4. Axial FR distributions in the far-lamp region $(60 \mathrm{~mm}$ from the sleeve surface) in various particle suspensions with various turbidities of (a) 2.0 NTU, (b) 10.0 NTU, and (c) 20.0 NTU.

MFSD was moved from the lamp center to the lamp end. In the central lamp region, the FR values of all three SOPs were similar for conditions of turbidity of $2.0 \mathrm{NTU}$; at this relatively low value of turbidity, the effects of suspended particles on the FR field were limited. For turbidities of 10.0 NTU and 20.0 NTU, the FR values for the $\mathrm{SiO}_{2}$ and $\mathrm{MgO}$ suspensions were higher than those of the $\mathrm{TiO}_{2}$ suspension at the same turbidity. These results were also consistent with the previously described scattering behavior of the particles (Figure $2 b$ and $c$ ). The axial FR distributions in the near-lamp region ( $5 \mathrm{~mm}$ from the sleeve surface) for each particle suspension are shown in Figure S1. Because of the short path-length, FR values of all three SOPs were similar in all turbidity cases. 
Mixed Particle Suspensions. The particles in practical waters come principally from soil (erosion). ${ }^{18,19}$ The composition of natural particles is variable and depends largely on local geology and land use practices. Natural particle suspensions likely comprise mixtures of silica and metal oxides, as well as organic materials. Hence, for the practical applications of UV photoreactors, it is relevant to examine the behavior of mixed particle suspensions involving mixtures of highly reflective (e.g., $\left.\mathrm{SiO}_{2}\right)$ and minimally reflective $\left(\mathrm{TiO}_{2}\right)$ particles. To address this issue, mixed suspensions of $\mathrm{TiO}_{2}$ and $\mathrm{SiO}_{2}$ particles were prepared. Radial FR distributions for mixtures of $\mathrm{SiO}_{2}$ and $\mathrm{TiO}_{2}$ with turbidity of $10.0 \mathrm{NTU}$ are shown in Figure 5. Turbidity contribution ratios of $\mathrm{SiO}_{2}$ to $\mathrm{TiO}_{2}$ were 7:3, 5:5,

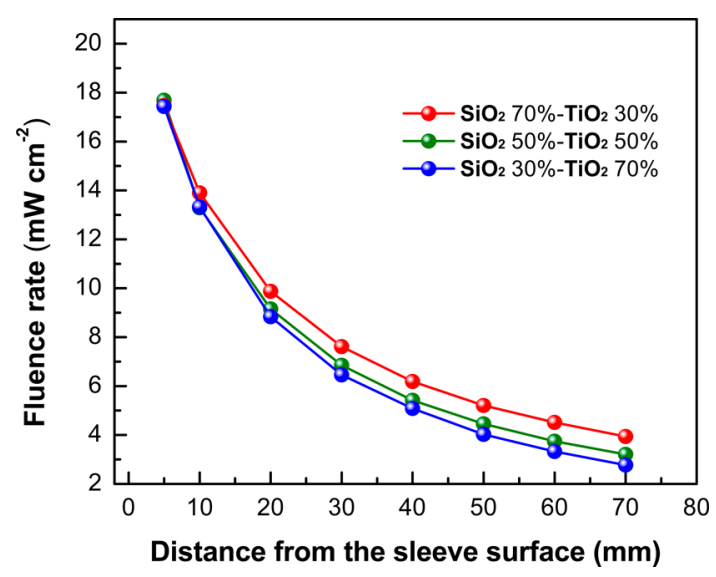

Figure 5. Radial FR distributions in mixed particle suspensions with turbidity of 10.0 NTU.

and 3:7. Because of the stronger scattering property of $\mathrm{SiO}_{2}$ as compared to that of $\mathrm{TiO}_{2}$, the FR distribution of the suspension with $\mathrm{SiO}_{2} 70 \%-\mathrm{TiO}_{2} 30 \%$ was higher than that of suspension with $\mathrm{SiO}_{2} 30 \%-\mathrm{TiO}_{2} 70 \%$, and the $\mathrm{FR}$ distribution of the suspension with $\mathrm{SiO}_{2} 50 \%-\mathrm{TiO}_{2} 50 \%$ was intermediate to the other two suspensions. Similar results were observed in the suspensions with turbidity of 20.0 NTU, as shown in SI Figure S2.

Weighted Average FR and Minimum FR. Table 2 and SI Table S1 illustrate the weighted average FR (WAFR) values

Table 2. Weighted Average Fluence Rates for Various Particle Suspensions

\begin{tabular}{lclll} 
& \multicolumn{3}{c}{ weighted average fluence rate $\left(\mathrm{mW} \mathrm{cm}^{-2}\right)$} \\
\cline { 2 - 5 } \multicolumn{1}{c}{ suspensions } & $0 \mathrm{NTU}$ & $2.0 \mathrm{NTU}$ & $10.0 \mathrm{NTU}$ & $20.0 \mathrm{NTU}$ \\
\hline deionized water & 5.824 & & & \\
$\mathrm{SiO}_{2}$ & & 6.120 & 6.386 & 7.784 \\
$\mathrm{TiO}_{2}$ & 6.278 & 4.870 & 3.979 \\
$\mathrm{MgO}$ & 5.973 & 6.244 & 6.454 \\
& $5.0 \mathrm{NTU}$ & $10.0 \mathrm{NTU}$ & $20.0 \mathrm{NTU}$ \\
$\mathrm{SiO}_{2} 30 \%-\mathrm{TiO}_{2} 70 \%$ & 5.911 & 4.890 & 3.996 \\
$\mathrm{SiO}_{2} 50 \%-\mathrm{TiO}_{2} 50 \%$ & N/A & 5.111 & 4.831 \\
$\mathrm{SiO}_{2} 70 \%-\mathrm{TiO}_{2} 30 \%$ & 5.985 & 5.314 & 5.213 \\
\end{tabular}

and the FR values at $70 \mathrm{~mm}$ distance to the sleeve surface in the central cross-section (regarded as the minimum FR) for DI water, as well as for the $\mathrm{SiO}_{2}, \mathrm{TiO}_{2}$, and $\mathrm{MgO}$ suspensions with various turbidity values. The WAFR values for the $\mathrm{SiO}_{2}$ suspensions with turbidity values of 2.0,10.0, and 20.0 NTU were $1.05,1.10$, and 1.34 times higher than those of DI water, respectively. Similarly, the minimum FR values for the $\mathrm{SiO}_{2}$ suspensions with turbidity values of 2.0,10.0, and 20.0 NTU were $1.02,1.12$, and 1.29 times higher than those in DI water, respectively. The minimum FR will be related to the minimum dose (or low-dose end of the dose distribution), which limits the performance of UV disinfection. ${ }^{28}$

The results reported herein, which were developed through the use of the MFSD, demonstrate that the presence of reflective suspended particles (i.e., particles that scatter UV photons) can actually enhance the FR field and the overall performance of a photoreactor. A previous study also demonstrated this enhancement effect. ${ }^{23}$ This seems contrary to the conventional view of UV photoreactors in the sense that turbidity is commonly regarded as an inhibiting factor for performance of these systems. In fact, the reflection by the particles could extend the photon path length in the reactor. ${ }^{23}$

The low photon energy loss during the absorption/reflection process on $\mathrm{SiO}_{2}$ particles (high UV reflection coefficient) could reduce interactions of $\mathrm{UV}$ photons with the reactor wall where they would be absorbed or inefficiently reflected, thereby enhancing WAFR in the reactor. Note that for UV disinfection, suspended particles can shield microbes from UV exposure, thereby counteracting the benefits described above. Therefore, the effects of suspended particles (turbidity) on the performance of UV disinfection systems can be quite complex. ${ }^{20-23,29}$

The WAFR values and minimum FR values for the $\mathrm{MgO}$ suspensions increased with increasing turbidity, and all WAFR values and minimum FR values for the $\mathrm{MgO}$ suspensions were higher than those for DI water. For each turbidity condition (i.e., 2.0, 10.0, or 20.0 NTU), because the $\mathrm{MgO}$ particles were less effective at scattering $\mathrm{UV}_{254}$ radiation than $\mathrm{SiO}_{2}$ particles (Figure 2c), its WAFR value and minimum $\mathrm{FR}$ values were lower than the corresponding values for the $\mathrm{SiO}_{2}$ suspension.

Calculated values of WAFR for the $\mathrm{TiO}_{2}$ suspensions dropped from 6.278 to $3.979 \mathrm{~mW} \mathrm{~cm}^{-2}$ as turbidity increased from 2.0-10.0 NTU. Meanwhile, the minimum FR value decreased from 4.01 to $1.95 \mathrm{~mW} \mathrm{~cm}^{-2}$ in the turbidity range of 2.0-10.0 NTU. At turbidity values of 10.0 and 20.0 NTU, the WAFR values were lower than that of DI water. At any given value of turbidity, the $\mathrm{TiO}_{2}$ suspensions had the highest transmittance (Figure $2 \mathrm{~b}$ ) and the lowest scattering at $254 \mathrm{~nm}$ (Figure 2c). Collectively, these attributes increase the likelihood of $\mathrm{UV}_{254}$ photons being incident on and absorbed by the reactor wall. Moreover, the low reflection coefficient of $\mathrm{TiO}_{2}$ powder at $254 \mathrm{~nm}$ (i.e., 0.135) implies that a large fraction of the UV photons were absorbed by the $\mathrm{TiO}_{2}$ particles in suspension. Therefore, unlike the $\mathrm{SiO}_{2}$ and $\mathrm{MgO}$ suspensions, an inhibition effect of $\mathrm{TiO}_{2}$ particles was observed.

Table 2 also illustrates WAFR calculations for mixed suspensions with various ratios of $\mathrm{SiO}_{2}$ to $\mathrm{TiO}_{2}$. At a fixed value of turbidity, WAFR increased with $\mathrm{SiO}_{2}$ content. Moreover, as turbidity increased, the WAFR values of all three mixed suspensions decreased, and the suspension with the highest $\mathrm{TiO}_{2}$ ratio demonstrated the most rapid decrease. In addition, all WAFR values of the mixed suspensions of $\mathrm{SiO}_{2}$ and $\mathrm{TiO}_{2}$ at fixed turbidity (5.0 NTU and 10.0 NTU) were greater than based on $\mathrm{TiO}_{2}$ alone, and lower than those of $\mathrm{SiO}_{2}$ alone.

Discussion. This study illustrated the impact of turbidity, a conventional water parameter used for characterizing the concentration of suspended particles in water, on the FR field of a simple UV photoreactor. The results demonstrate that several optical properties of suspended particles can induce the 
FR distribution in a UV reactor at an identical turbidity value. This is explained by the fact that conventional measurements of turbidity involve visible radiation, whereas the optical properties of particles in the UVC range are perhaps more relevant to the behavior of a UV photoreactor. Moreover, differences in particle composition are associated with differences in optical behavior in the visible and UVC ranges. Therefore, conventional turbidity measurements (based on visible light) are inappropriate for characterization of the effects of suspended particles on the FR field. As alternatives, measurements of reflectance and scattering at the characteristic wavelength of the UV source are more representative of the effects of suspended particles on the FR field.

The measurements reported herein demonstrate that the optical field (FR distribution or WAFR) was principally dependent on the photon scattering ability of the suspension, which corresponded with measurements of photon absorption and reflection by the solid powders in the suspension (reflection coefficient), as well as other factors such as the particle size and shape. ${ }^{29}$ For suspensions with identical turbidity, particles that absorb poorly in a UVC wavelength range (e.g., $\mathrm{SiO}_{2}$ and $\mathrm{MgO}$ ) could enhance the FR distribution and performance of a photoreactor. Conversely, for particles that absorb strongly in UVC range (e.g., $\mathrm{TiO}_{2}$ ), their presence in suspension could diminish the FR distribution. Meanwhile, the turbidity value could represent the particle concentration in the suspensions, implying that the increasing turbidity could amplify the effect of the particles on the FR distribution in the reactor, including positive effects (e.g., $\mathrm{SiO}_{2}$ and $\mathrm{MgO}$ ), and negative effects (e.g., $\mathrm{TiO}_{2}$ ).

The effects of suspended particles on the optical behavior of UV radiation were also characterized by measurements of transmittance and scattering intensity developed using a spectrometer and a fluorescence spectrometer, respectively. These two tests have the potential to be used as characterization methods for evaluating the effects of turbidity on FR distributions.

\section{ASSOCIATED CONTENT}

\section{S Supporting Information}

The Supporting Information is available free of charge on the ACS Publications website at DOI: 10.1021/acs.est.7b02730.

Two figures and one table are provided. Axial FR distributions in the near-lamp region ( $5 \mathrm{~mm}$ from the sleeve surface) with various particle suspensions with various turbidities (Figure S1), radial FR distributions with mixed particle suspensions with various turbidities of 20.0 NTU (Figure S2), and minimum FR values (Table S1) (PDF)

\section{AUTHOR INFORMATION}

\section{Corresponding Authors}

*(E.R.B.) Phone: 1-765-494-0316; fax: 1-765-494-0395; e-mail: blatch@purdue.edu.

*(Z.Q.) Phone: +86 10 62849632; fax: +86 10 62923541; email: qiangz@rcees.ac.cn.

\section{ORCID $\odot$}

Zhimin Qiang: 0000-0002-6555-3381

Ernest R. Blatchley III: 0000-0002-4561-8635

Notes

The authors declare no competing financial interest.

\section{ACKNOWLEDGMENTS}

We gratefully acknowledge the financial support from the National Natural Science Foundation of China (51408592, 21590814, 51525806), Edward M. Curtis Visiting Professorship of Purdue University, and National Geographic Air and Water Conservation Fund (GERC23-15).

\section{REFERENCES}

(1) Rizzo, L.; Della Sala, A.; Fiorentino, A.; Li Puma, G. Disinfection of urban wastewater by solar driven and UV lamp - $\mathrm{TiO}(2)$ photocatalysis: Effect on a multi drug resistant Escherichia coli strain. Water Res. 2014, 53, 145-52.

(2) Zhao, Q.; Shang, C.; Zhang, X.; Ding, G.; Yang, X. Formation of halogenated organic byproducts during medium-pressure UV and chlorine coexposure of model compounds, NOM and bromide. Water Res. 2011, 45 (19), 6545-54.

(3) Duran, J. E.; Taghipour, F.; Mohseni, M. Evaluation of model parameters for simulating $\mathrm{TiO}(2)$ coated UV reactors. Water Sci. Technol. 2011, 63 (7), 1366-72.

(4) Bagheri, M.; Mohseni, M. Computational fluid dynamics (CFD) modeling of VUV/UV photoreactors for water treatment. Chem. Eng. J. 2014, 256, 51-60.

(5) Taghipour, F.; Sozzi, A. Modeling and design of ultraviolet reactors for disinfection by-product precursor removal. Desalination 2005, 176 (1-3), 71-80.

(6) Sozzi, D. A.; Taghipour, F. UV reactor performance modeling by Eulerian and Lagrangian methods. Environ. Sci. Technol. 2006, 40 (5), $1609-1615$

(7) Jin, S. S.; Linden, K. G.; Ducoste, J.; Liu, D. Impact of lamp shadowing and reflection on the fluence rate distribution in a multiple low-pressure UV lamp array. Water Res. 2005, 39 (12), 2711-2721.

(8) Jacob, S. M.; Dranoff, J. S. Light intensity profiles in a perfectly mixed photoreactor. AIChE J. 1970, 16 (3), 359-366.

(9) Blatchley, E. R., III Numerical modelling of UV intensity: Application to collimated-beam reactors and continuous-flow systems. Water Res. 1997, 31 (9), 2205-2218.

(10) Irazoqui, H. A.; Cerda, J.; Cassano, A. E. Radiation profiles in an empty annular photoreactor with a source of finite spatial dimensions. AIChE J. 1973, 19 (3), 460-467.

(11) Rahn, R. O.; Bolton, J.; Stefan, M. I. The iodide/iodate actinometer in UV disinfection: determination of the fluence rate distribution in UV reactors. Photochem. Photobiol. 2006, 82 (2), 611615 .

(12) Li, M. K.; Qiang, Z.; Li, T. G.; Bolton, J. R.; Liu, C. L. In situ measurement of UV fluence rate distribution by use of a micro fluorescent silica detector. Environ. Sci. Technol. 2011, 45 (7), 30343039.

(13) Bolton, J. R. Calculation of ultraviolet fluence rate distributions in an annular reactor: Significance of refraction and reflection. Water Res. 2000, 34 (13), 3315-3324.

(14) Li, M. K.; Qiang, Z. M.; Wang, C.; Bolton, J. R.; Blatchley, E. R., III Experimental assessment of photon fluence rate distributions in a medium-pressure UV photoreactor. Environ. Sci. Technol. 2017, 51 (6), $3453-3460$.

(15) Bohren, C. F.; Nevitt, T. J. Absorption by a sphere: a simple approximation. Appl. Opt. 1983, 22 (6), 774-775.

(16) Qualls, R. G.; Johnson, J. D. Bioassay and dose measurement in UV disinfection. Appl. Environ. Microbiol. 1983, 45 (3), 872-877.

(17) American Public Health Association Standard Methods for the Examination of Water and Wastewater, 14th ed., American Public Health Association, New York, 1975.

(18) Davis, M. L.; Cornwell, D. A. Introduction to Environmental Engineering, 4th ed., McGraw-Hill, New York, 2008.

(19) Mihelcic, J. R.; Zimmerman, J. B. Environmental Engineering: Fundamentals, Sustainability, Design, 2nd ed.; John Wiley \& Sons, Hoboken, NJ. 
(20) Passantino, L.; Malley, J.; Knudson, M.; Ward, R.; Kim, J. Effect of low turbidity and alage on UV disinfection performance. J. Am. Water Works Assoc. 2004, 96 (6), 123-137.

(21) Amoah, K.; Craik, S.; Smith, D. W.; Belosevic, M. Inactivation of Cryptosporidium oocysts and Giardia cysts by ultraviolet light in the presence of natural particulate matter. J. Water Supply Res. Technol. Aqua 2005, 54, 165-178.

(22) Malley, R.; Vernacchio, L.; Devincenzo, J.; Ramilo, O.; Dennehy, P. H.; Meissner, H. C.; Gruber, W. C.; Jafri, H. S.; Sanchez, P. J.; Macdonald, K.; Montana, J..

(23) Templeton, M. R.; Andrews, R. C.; Hofmann, R. Inactivation of particle-associated viral surrogates by ultraviolet light. Water Res. 2005, 39 (15), 3487-3500.

(24) Mamane, H.; Ducoste, J. J.; Linden, K. G. Effect of particles on ultraviolet light penetration in natural and engineered systems. Appl. Opt. 2006, 45 (8), 1844-1856.

(25) Li, M. K.; Qiang, Z. M.; Bolton, J. R.; Ben, W. W. Impact of reflection on the fluence rate distribution in a UV reactor with various inner walls as measured using a micro-fluorescent silica detector. Water Res. 2012, 46 (11), 3595-3602.

(26) Li, M. K.; Qiang, Z. M.; Bolton, J. R. In situ detailed fluence rate distributions in a UV reactor with multiple low-pressure lamps: Comparison of experimental and model results. Chem. Eng. J. 2013, 214, 55-62.

(27) Cao, Y.; Feng, J.; Wu, P. Preparation of organically dispersible graphene nanosheet powders through a lyophilization method and their poly (lactic acid) composites. Carbon 2010, 48 (13), 3834-3839. (28) Chiu, K.; Lyn, D. A.; Savoye, P.; Blatchley, E. R., III Integrated UV disinfection model based on particle tracking. J. Environ. Eng. 1999, 125 (1), 7-16.

(29) Mamane, H. Impact of particles on UV disinfection of water and wastewater effluents: A review. Rev. Chem. Eng. 2008, 24 (2-3), 67157. 